\title{
A Comparative Analysis of the Safety Profile of Direct Oral Anticoagulants Using the FDA Adverse Event Reporting System
}

\author{
Emma P. DeLoughery ${ }^{1}$, Joseph J. Shatzel, MD² \\ ${ }^{1}$ Mayo Clinic School of Medicine, Mayo Clinic, Rochester MN, \\ ${ }^{2}$ Division of Hematology, Oregon Health \& Science University, Portland OR
}

\begin{abstract}
Introduction: Direct oral anticoagulants (DOACs) are being increasingly used. However, unlike warfarin, less is known regarding their long-term side effects. To better evaluate the rates of DOAC-related adverse events (AEs) on a population level we examined AEs reported to the FDA for three commonly used DOACs and warfarin.
\end{abstract}

Methods: We evaluated the FDA Adverse Event Reporting System (FAERS) database, which compiles reported drug-related AEs from 1969 onwards. The safety profiles of the included drugs were assessed by comparing AEs per outpatient prescription and with proportional reporting ratios (PRR).

Results: Rivaroxaban had the highest proportion of reported AEs. Most notably the rate for breakthrough venous thromboembolism (VTE) was higher than other DOACs. Dabigatran had the highest reported rated of ischemic stroke. When the DOAC data was analyzed using PRR, reported rates of VTE were again higher with rivaroxaban while dabigatran again showed slightlyhigher than expected rates of ischemic stroke. Apixaban did not show higher than expected rates in any category.

Conclusion: Our analysis found rates of reported breakthrough VTE were significantly higher with rivaroxaban, while apixaban had no higher than expected rates of any studied AEs.

\section{Introduction:}

As we approach a decade with direct oral anticoagulants (DOACs), increasing information on their safety and efficacy has become available. As compared with warfarin, numerous studies have shown equivalent or improved efficacy and safety with DOACs, which combined with their decreased restrictions on lifestyle make them an increasingly popular alternative ${ }^{1,2}$. However, unlike the century-old warfarin, less is known regarding the longterm and large-scale side effects of DOACs, nor is there sufficient data to define the comparative safety among the available DOACs. Some suggestion of the rates of DOACassociated adverse events on a population-level can be found in the U.S. Food \& Drug Administration (FDA) Adverse Event Reporting System (FAERS), which compiles adverse

\footnotetext{
Correspondence to: Emma P. DeLoughery, $2001^{\text {st }}$ St. SW, Rochester MN, 55905, deloughery.emma@mayo.edu.
}

Conflict of interest statement: The authors have no conflicts of interest 
events reported to the FDA from 1969 onwards. Adverse events refer to any event experienced by the patient while taking the medication and may not necessarily be related to the drug. FAERS supports post-marketing surveillance, allowing for faster and clearer linkage between drugs and adverse events than would be possible in smaller trials ${ }^{3}$. While it does have limitations such as being reliant upon events being reported, potential for duplication of reports, and discrepancies in labeling adverse events, it provides a look into the range and frequency of adverse events reported for a specific drug. In this study, select hematologic adverse events were compiled for warfarin and three DOACs and events per prescription rates were generated in order to compare event rates across the anticoagulant spectrum.

\section{Methods:}

The FDA Adverse Event Reporting System (FAERS) is a United States federal database containing adverse event reports, medication error reports and product quality complaints submitted to FDA. The database is designed to support the FDA's post-marketing safety surveillance program. Adverse events listed in FAERS result from voluntary reports directly from healthcare providers(such as physicians, pharmacists, nurses and others) and consumers (such as patients, family members, lawyers and others). ${ }^{4}$

Adverse event data for each included drug (warfarin, dabigatran, rivaroxaban, apixaban) was gathered from FAERS. Edoxaban was not included in this analysis given its limited use in the US, and Betrixaban was not included given it was only recently FDA approved. ${ }^{5,6}$. The generic name of each drug was used, and in the case of dabigatran and warfarin, dabigatran etexilate mesylate and warfarin sodium were used respectively to query the database. For each drug included in the study, FAERS includes a list of adverse events. The events of interest were extracted by a single author (ED). Adverse events data was filtered by including only those reported by healthcare professionals in the United States. Specific adverse events were chosen apriori based on hypothesized potential complications and included hemorrhagic and thrombotic complications. Gastrointestinal (GI) hemorrhage and intracranial hemorrhage (ICH) were hypothesized hemorrhagic complications likely due to direct side effects of the anticoagulants, while deep vein thrombosis (DVT), pulmonary embolism (PE), myocardial infarction (MI), and ischemic stroke were potential complications due to the underlying disease process and possible under-treatment.

Numbers of U.S. outpatient prescriptions were gathered from the ClinCalc DrugStats database using information from the Medical Expenditure Panel Survey (MEPS).

Only adverse events data from years in which there was also prescription data were included in the study. Comparisons of total adverse events per prescription were from 2004-2015 for warfarin, 2010-2015 for dabigatran, 2012-2015 for rivaroxaban, and 2014-2015 for apixaban.

The proportional reporting ratio (PRR) with 95\% confidence interval was used to compare rates of reactions among the DOACs, with warfarin being excluded to avoid the new-drug effect. Given that warfarin's side effects are well-known and well-characterized, it is 
unlikely that an expected complication of using warfarin would be reported to the FDA. Thus, including warfarin in an analysis reliant upon all drugs having the same baseline probability of having an adverse event reported would artificially skew the results towards warfarin having a better safety profile simply due to its adverse events likely not being reported.

The PRR compares the rate of an event occurring in the drug of interest to the rate of the event occurring in all other drugs in the study, with a value equal to or greater than 2 often being considered significant ${ }^{7}$. The PRR for rivaroxaban and DVT, for example, would be calculated as the rate of DVT among rivaroxaban cases divided by the rate of DVT among cases for all other drugs in the study. Total cases - each representing one patient - rather than total number of adverse events was used as the denominator for calculations as one case might include reports ofmultiple adverse events. A significance level of 2 with 95\% confidence interval was used to assess for statistical significance.

\section{Results:}

A total of 42,964 cases were included in the study: 10,909 on warfarin, 10,850 on dabigatran, 17,962 on rivaroxaban, and 3243 on apixaban. Of all four anticoagulants, warfarin had the lowest rate per prescription for each adverse event. In contrast, rivaroxaban often had the highest rate for each event. Rivaroxaban had the highest rate per prescription of cases overall, as well as the highest reported rate of death, GI hemorrhage, ICH, PE, DVT, MI. Dabigatran had the highest reported rate of ischemic stroke (Table 1).

The PRR was used to compare adverse event rates for death, GI hemorrhage, ICH, PE, DVT, MI, and ischemic stroke, as seen in Figure 1. The PRR for death ( 0.90 dabigatran vs. 1.04 rivaroxaban vs. 1.16 apixaban) GI hemorrhage ( 1.23 dabigatran vs. 0.97 rivaroxaban vs. 0.57 apixaban) and ICH ( 0.40 dabigatran vs. 1.04 rivaroxaban vs. 1.08 apixaban) did not differ significantly among DOACs (Table 1). Rivaroxaban had a significant association with reports for PE ( 0.29 dabigatran vs. 3.68 rivaroxaban vs. 0.44 apixaban) and DVT (0.28 dabigatran vs. 3.24rivaroxaban vs. 0.65 apixaban). Dabigatran had the highest, though nonsignificant, association with MI (1.73 dabigatran vs. 0.73 rivaroxaban vs. 0.46 apixaban) and ischemic stroke (2.41dabigatran vs. 0.47 rivaroxaban vs. 0.72 apixaban).

\section{Discussion:}

Our analysis of over 40,000 oral anticoagulant related adverse event cases reported to the FDA found rivaroxaban had a numerically higher rate of the included adverse events, and a significantly higher proportional reporting ratio for breakthrough venous thromboembolism. Our findings are parallel to prior work using FAERS to assess DOAC safety ${ }^{89}$ some of which found disproportionate safety reports with rivaroxaban ${ }^{3}$. Our findings also parallel multiple large observational studies that comparatively evaluate DOACs, several of which have found increased thromboembolic events and bleeding with rivaroxaban as compared with other oral anticoagulants. ${ }^{10,11}$ Other analyses have suggested an increased rate of cardiovascular events associated with dabigatran, in line with the findings of this study. ${ }^{12}$ 
Rivaroxaban is unique among the included DOACs given its once-per-day dosing for the treatment of both atrial fibrillation and venous thromboembolism ${ }^{3}$. This once-daily dosing leads to distinct pharmacokinetics and pharmacodynamics, which may explain the unexpectedly high proportion of thromboembolic reported with rivaroxaban. Apixaban and dabigatran are both twice-daily drugs, which may result in decreased variability in blood levels and a more constant anticoagulant effect, potentially sparing some hemorrhagic and thrombotic complications ${ }^{1}$. Reduced dosing has shown equivalent efficacy in secondary VTE prevention for apixaban and later for rivaroxaban, however it is unclear if these lower doses correlate to a lower risk of bleeding, and howmuch different dosing effects affected adverse event reporting ${ }^{13}$.

While our results are in line with prior studies, this analysis has some limitations, including the shortcomings inherent to the FAERS and MEPS databases. FAERS includes a large number of possible reactions, many of which overlap, which could have led to under- or over-reporting of reactions. For example, if a patient on dabigatran had a DVT, it could be reported in FAERS as a DVT and/or a thrombosis, which could have led to double-counting. However, it is likely that given the large number of cases for all drugs, such under- and overcounting scenarios would tend to balance out. Secondly, correlation is not causation, and that a patient, one of whose medications included rivaroxaban, had an MI does not mean that rivaroxaban was the cause of that MI.he FAERS data displays general trends only. Likewise, biases that affect the PRR include that it primarily measures differences in reports of adverse events, which may or may not correlate to the occurrence of adverse events ${ }^{14}$. Not every adverse event that occurs with a drug is reported, nor is the clinical legitimacy of each event confirmed. Many factors can influence whether an event will be reported to the FDA, such as the time a product has been marketed and publicity about specific potential adverse events. Given all of the potential for bias, FAERS data cannot be used to report true incidence rates for adverse events or comparative differences in clinical effectiveness. Never the less, the data is hypothesis generating and appears to correlate with other reports of the included DOACs. Also, the MEPS database represents survey data that may or may not be representative of the true number of prescriptions dispensed. Finally, while 6 years of data was available for dabigatran, only 4 years of rivaroxaban and 2 years of apixaban data were available. Therefore, particularly for apixaban, trends over time in reported adverse events were not able to be seen, and it is possible that different patterns will emerge with future data.

In conclusion, our analysis found rivaroxaban has a high rate of reported hemorrhagic and thrombotic events per prescription, and a significantly higher reported rate of breakthrough VTE in comparison with dabigatran and apixaban. Of the DOACs, apixaban appears to offer the best safety profile using this data, which is consistent with prior observational studies, ${ }^{11}$ though more time and more data is needed to offer a definitive answer as to the safest anticoagulant.

\section{References}

1. Lim HY, Nandurkar H, Ho P. Direct Oral Anticoagulants and the Paradigm Shift in the Management of Venous Thromboembolism. Seminars in thrombosis and hemostasis. 2018;44(3):261-266. [PubMed: 29558778] 
2. Hernandez I, Zhang Y, Saba S. Effectiveness and Safety of Direct Oral Anticoagulants and Warfarin, Stratified by Stroke Risk in Patients With Atrial Fibrillation. The American journal of cardiology. 2018.

3. Alshammari TM, Ata SI, Mahmoud MA, Alhawassi TM, Aljadhey HS. Signals of bleeding among direct-acting oral anticoagulant users compared to those among warfarin users: analyses of the postmarketing FDA Adverse Event Reporting System (FAERS) database, 2010-2015. Therapeutics and clinical risk management. 2018;14:803-809. [PubMed: 29750038]

4. https://www.fda.gov/drugs/guidancecomplianceregulatoryinformation/surveillance/ adversedrugeffects/default.Htm. Accessed March 2019.

5. Skelley JW, Thomason AR, Nolen JC, Candidate P. Betrixaban (Bevyxxa): A Direct-Acting Oral Anticoagulant Factor Xa Inhibitor. P \& T : a peer-reviewed journal for formulary management. 2018;43(2):85-120. [PubMed: 29386864]

6. http://www.cardiobrief.org/2016/10/20/eliquis-nipping-at-the-heals-of-noac-market-leader-xarelto/. Accessed December, 2018.

7. Evans SJ, Waller PC, Davis S. Use of proportional reporting ratios (PRRs) for signal generation from spontaneous adverse drug reaction reports. Pharmacoepidemiology and drug safety. 2001;10(6):483-486. [PubMed: 11828828]

8. Nathan KT, Conn KM, van Manen RP, Brown JE. Signal detection for bleeding associated with the use of direct oral anticoagulants. American journal of health-system pharmacy : AJHP : official journal of the American Society of Health-System Pharmacists. 2018.

9. Serebruany V, Cherepanov V, Fortmann S, Kim MH. Mortality and oral anticoagulants in the Food and Drug Administration Adverse Event Reporting System. Open heart. 2017;4(2):e000629. [PubMed: 28761683]

10. Yao X, Abraham Neena S, Sangaralingham Lindsey R, et al. Effectiveness and Safety of Dabigatran, Rivaroxaban, and Apixaban Versus Warfarin in Nonvalvular Atrial Fibrillation. Journal of the American Heart Association.5(6):e003725. [PubMed: 27412905]

11. Deitelzweig S, Keshishian A, Li X, et al. COMPARISON OF EFFECTIVENESS, SAFETY, AND THE NET CLINICAL OUTCOME BETWEEN DIFFERENT DIRECT ORAL ANTICOAGULANTS IN 162,707 NON-VALVULAR ATRIAL FIBRILLATION PATIENTS TREATED IN US CLINICAL PRACTICE. Journal of the American College of Cardiology. 2018;71(11 Supplement):A275.

12. Douxfils J, Buckinx F, Mullier F, et al. Dabigatran Etexilate and Risk of Myocardial Infarction, Other Cardiovascular Events, Major Bleeding, and All-Cause Mortality: A Systematic Review and Meta-analysis of Randomized Controlled Trials. Journal of the American Heart Association. 2014;3(3):e000515. [PubMed: 24906369]

13. Vasanthamohan L, Boonyawat K, Chai-Adisakopha C, Crowther M. Reduced dose direct oral anticoagulants in the extended treatment of venous thromboembolism: a systematic review and meta-analysis. Journal of thrombosis and haemostasis : JTH. 2018.

14. Moore N, Hall G, Sturkenboom M, Mann R, Lagnaoui R, Begaud B. Biases affecting the proportional reporting ratio (PPR) in spontaneous reports pharmacovigilance databases: the example of sertindole. Pharmacoepidemiology and drug safety. 2003;12(4):271-281. [PubMed: 12812006] 


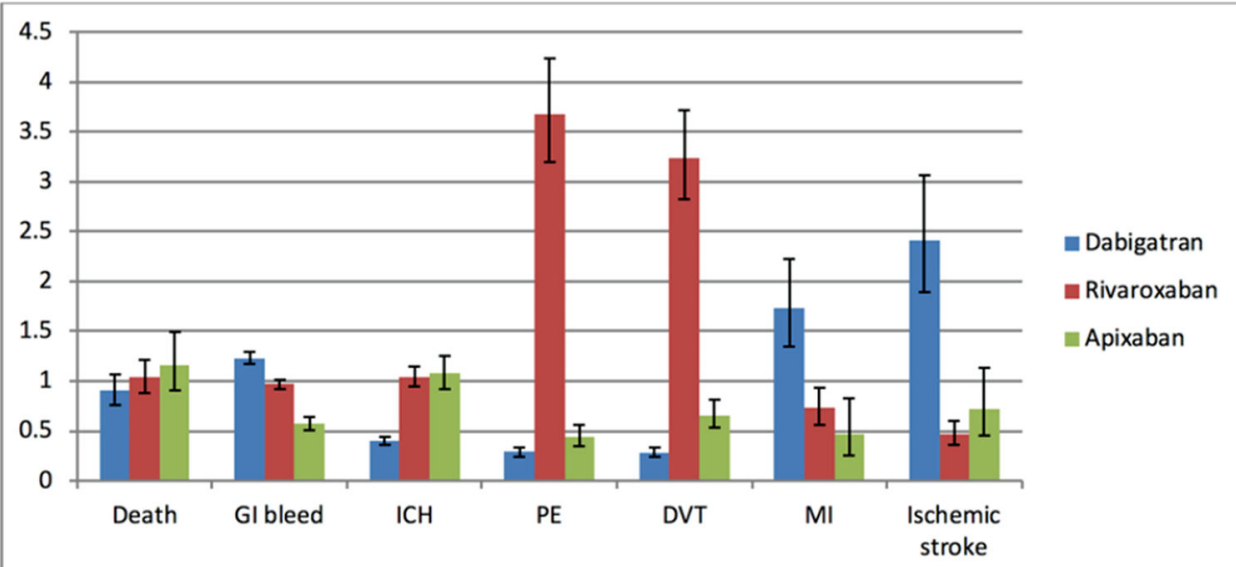

Figure 1:

Proportional reporting ratio for DOACs 
Table 1:

Events per outpatient prescription and proportional reporting ratio for warfarin and DOACs

\begin{tabular}{|c|c|c|c|c|c|c|c|}
\hline & Warfarin & Dabigatran & PRR & Rivaroxaban & PRR & Apixaban & PRR \\
\hline Cases & 10909 & 10850 & & 17962 & & 3243 & \\
\hline Prescriptions & 323584946 & 13236206 & & 11977071 & & 4636173 & \\
\hline Death & $135\left(4.17 * 10^{-7}\right)$ & $183\left(1.38 * 10^{-5}\right)$ & $\begin{array}{c}0.90(0.76- \\
1.07)\end{array}$ & $330\left(2.76^{*} 10^{-5}\right)$ & $\begin{array}{c}1.04(0.88- \\
1.22)\end{array}$ & $67\left(1.45^{*} 10^{-5}\right)$ & $\begin{array}{c}1.16(0.90- \\
1.49)\end{array}$ \\
\hline GI bleed & $\begin{array}{c}1107 \\
\left(3.42 * 10^{-6}\right)\end{array}$ & $2036\left(1.54 * 10^{-4}\right)$ & $\begin{array}{c}1.23(1.17- \\
1.30)\end{array}$ & $2910\left(2.43 * 10^{-4}\right)$ & $\begin{array}{c}0.97(0.92- \\
1.02)\end{array}$ & $318\left(6.86 * 10^{-5}\right)$ & $\begin{array}{c}0.57(0.51- \\
0.64)\end{array}$ \\
\hline $\mathrm{ICH}$ & $433\left(1.34 * 10^{-6}\right)$ & $530\left(4.00 * 10^{-5}\right)$ & $\begin{array}{c}0.40(0.36- \\
0.44)\end{array}$ & $941\left(7.86 * 10^{-5}\right)$ & $\begin{array}{c}1.04(0.95- \\
1.15)\end{array}$ & $178\left(3.84 * 10^{-5}\right)$ & $\begin{array}{c}1.08(0.92- \\
1.25)\end{array}$ \\
\hline PE & $202\left(6.24 * 10^{-7}\right)$ & $169\left(1.28 * 10^{-5}\right)$ & $\begin{array}{c}0.29(0.25- \\
0.34)\end{array}$ & $1084\left(9.05 * 10^{-5}\right)$ & $\begin{array}{c}3.68(3.20- \\
4.24)\end{array}$ & $62\left(1.34 * 10^{-5}\right)$ & $\begin{array}{c}0.44(0.34- \\
0.57)\end{array}$ \\
\hline DVT & $263\left(8.13 * 10^{-7}\right)$ & $162\left(1.22 * 10^{-5}\right)$ & $\begin{array}{c}0.28(0.24- \\
0.33)\end{array}$ & $1033\left(8.63 * 10^{-5}\right)$ & $\begin{array}{c}3.24(2.83- \\
3.72)\end{array}$ & $88\left(1.90 * 10^{-5}\right)$ & $\begin{array}{c}0.65(0.53- \\
0.81)\end{array}$ \\
\hline MI & $67\left(2.07 * 10^{-7}\right)$ & $114\left(8.61 * 10^{-6}\right)$ & $\begin{array}{c}1.73(1.34- \\
2.22)\end{array}$ & $117\left(9.77^{*} 10^{-6}\right)$ & $\begin{array}{c}0.73(0.57- \\
0.94)\end{array}$ & $12\left(2.59 * 10^{-6}\right)$ & $\begin{array}{c}0.46(0.26- \\
0.82)\end{array}$ \\
\hline Ischemic stroke & $20\left(6.18^{*} 10^{-8}\right)$ & $148\left(1.12^{*} 10^{-5}\right)$ & $\begin{array}{c}2.41(1.90- \\
3.06)\end{array}$ & $100\left(8.35^{*} 10^{-6}\right)$ & $\begin{array}{c}0.47(0.36- \\
0.60)\end{array}$ & $20\left(4.31 * 10^{-6}\right)$ & $\begin{array}{c}0.72(0.45- \\
1.13)\end{array}$ \\
\hline
\end{tabular}

(events per outpatient prescription) or (95\% confidence interval) 